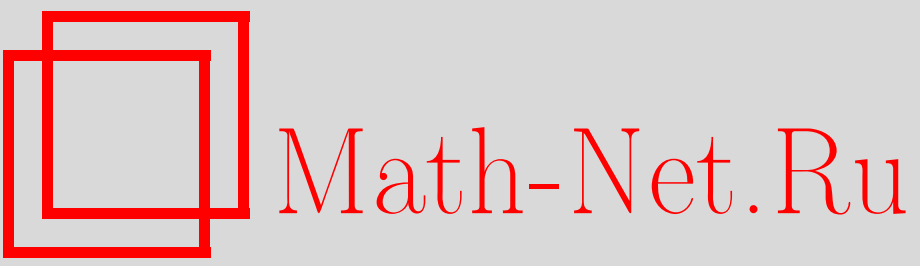

И. А. Дынников, Трехстраничное представление зацеплений, УМН, 1998, том 53, выпуск 5, 237-238

DOI: https://doi.org/10.4213/rm79

Использование Общероссийского математического портала Math-Net.Ru подразумевает, что вы прочитали и согласны с пользовательским соглашением

http://www.mathnet.ru/rus/agreement

Параметры загрузки:

IP : 54.92 .164 .108

26 апреля 2023 г., $17: 41: 52$

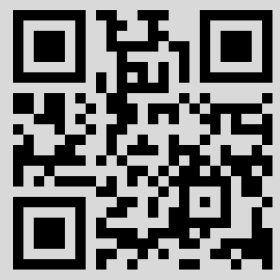




\section{ТРЕХСТРАНИЧНОЕ ПРЕДСТАВЛЕНИЕ ЗАЦЕПЛЕНИЙ}

\section{И. А. Дынников}

Рассмотрим в 3-мерном пространстве $\mathbb{R}^{3}$ объединение $\mathbb{Y}$ трех попарно различных полуплоскостей $P_{1}, P_{2}, P_{3}$ (страниц), имеющих общую граничную прямую (линию переплета): $\partial P_{1}=$ $\partial P_{2}=\partial P_{3}=l$, которая ориентирована с помощью направляющего вектора $\bar{\nu}$. Tp ехстраничной диаграмой зацепления мы назьваем произвольную несамопересекающуюся замкнутую ломаную $L \subset \mathbb{Y}$ (имеющую, возможно, несколько компонент связности) такую, что для любого $i \in \mathbb{Z}_{3}$ каждая связная компонента пересечения $L \cap P_{i}$ (арка) состоит из двух прямолинейных отрезков равной длины, перпендикулярных друг другу. Точки пересечения $L \cap l$ мы обозначаем через $M_{1}, \ldots, M_{n(L)}$ и нумеруем их в соответствии с ориентацией линии переплета $l$.

Каждую трехстраничную диаграмму зацепления мы будем кодировать некоторым словом в 12 -буквенном алфавите $\mathbb{A}=\left\{a_{i}, b_{i}, c_{i}, d_{i}\right\}_{i \in \mathbb{Z}_{3}}$. Для этого определим сначала отображение $\varphi=$ $\left(\varphi^{1}, \varphi^{2}, \varphi^{3}\right): \mathbb{A} \rightarrow \mathbb{Z}^{3}$ по формулам: $\varphi^{i}\left(a_{j}\right)=\delta_{j+1}^{i}+\delta_{j-1}^{i}, \varphi^{i}\left(b_{j}\right)=\delta_{j+1}^{i}-\delta_{j-1}^{i}, \varphi^{i}\left(c_{j}\right)=$ $-\delta_{j+1}^{i}-\delta_{j-1}^{i}, \varphi^{i}\left(d_{j}\right)=-\delta_{j+1}^{i}+\delta_{j-1}^{i}$, где $i, j \in \mathbb{Z}_{3}$, и $\delta_{i}^{j}$ есть символ Кронекера. $\mathrm{K}$ каждой точке $M_{p} \in L \cap l$ примькает два звена, скажем, $M_{p} Q$ и $M_{p} R$, ломаной $L$. Без ограничения общности можно считать, что $Q \in P_{i}, R \in P_{i+1}$, где $i \in \mathbb{Z}_{3}$. Мы говорим, что вершина $M_{p}$ имеет тип $x \in \mathbb{A}$, если $\varphi^{i}(x)=\operatorname{sgn}\left\langle\overline{M_{p} Q}, \bar{\nu}\right\rangle, \varphi^{i+1}(x)=\operatorname{sgn}\left\langle\overline{M_{p} R}, \bar{\nu}\right\rangle, \varphi^{i-1}(x)=0$, где $\langle\cdot, \cdot\rangle$ обозначает стандартное скалярное произведение в $\mathbb{R}^{3}$. Диаграме $L$ мы сопоставляем слово $w_{L}=$ $x_{1} \cdots x_{n(L)}$, если для всех $p \leqslant n(L)$ вершина $M_{p} \in L \cap l$ имеет тип $x_{p} \in \mathbb{A}$.

Нетрудно видеть, что по слову $w_{L}$ диаграмма $L$ может быть восстановлена однозначно с точностью до изотопии в $\mathbb{Y}$. Понятно также, что отнюдь не любое слово кодирует некоторую трехстраничную диаграмму. Слова в алфавите $\mathbb{A}$, которые имеют вид $w_{L}$, где $L-$ некоторая трехстраничная диаграмма, мы называем сбалансированными.

Рассмотрим теперь полугруппу $S$, порожденную множеством $\mathbb{A}$ и соотношениями

$$
\begin{gathered}
a_{i}=a_{i+1} d_{i-1}, \quad b_{i}=a_{i-1} c_{i+1}, \quad c_{i}=b_{i-1} c_{i+1}, d_{i}=a_{i+1} c_{i-1} \\
d_{1} d_{2} d_{3}=1, \\
b_{i} d_{i}=d_{i} b_{i}=1, \\
v w=w v, \\
(3) \\
\text { (4) } \quad \text { где } v \in\left\{a_{i-1} b_{i-1}, d_{i-1} c_{i-1}, b_{i+1} d_{i-1} d_{i+1} b_{i-1}\right\}, w \in\left\{a_{i}, b_{i}, c_{i}, b_{i-1} d_{i} d_{i-1}\right\},
\end{gathered}
$$

$i \in \mathbb{Z}_{3}$ (всего имеется 55 соотношений, из которых одно является следствием остальных). Для слова $w$ в алфавите $\mathbb{A}$ мы обозначаем через $[w]$ элемент полугруппы $S$, соответствующий слову $w$. мой;

ТЕОРема 1. 1) Любое зацепление можно представить трехстраничной диаграм-

2) две трехстраничные диаграммы $L_{1}$ и $L_{2}$ изотопны в $\mathbb{R}^{3}$ тогда и только тогда, когда $\left[w_{L_{1}}\right]=\left[w_{L_{2}}\right]$;

3) әлемент $s \in S$ может быть представлен сбалансированным словом (т.е. задает некоторое зачепление в $\mathbb{R}^{3}$ ) тогда и только тогда, когда он чентральный.

Таким образом, мы построили взаимно-однозначное соответствие между изотопическими классами неориентированных неупорядоченных зацеплений в $\mathbb{R}^{3}$ и центральньми элементами полугруппы $S$. Единице полугруппы при этом соответствует пустое зацепление $\varnothing$, а умножение в центре полугруппы соответствует взятию объединения зацеплений.

Tеорема 2. Группа $G$ обратимых әлементов полугруппь $S$ порождается образующими $d_{1}, d_{2}, d_{3}$ и соотношениями

$$
\begin{gathered}
d_{1} d_{2} d_{3}=1, \\
d_{i} u_{i}=u_{i} d_{i}, \\
u_{i} u_{j}=u_{j} u_{i},
\end{gathered}
$$

Работа выполнена при поддержке Российского фонда фундаментальных исследований (грант № 96-01-01404). 
где $u_{i}=d_{i+1}^{-1} d_{i-1} d_{i+1} d_{i-1}^{-1}, i, j \in \mathbb{Z}^{3}$.

Коммутант группь $G$ изоморфен бесконечной группе кос $B$.

Пусть $G_{i} \subset G$ - подгруппа, порожденная әлементами $d_{i} u u_{i+1}\left(=d_{i}^{2} u_{i-1} d_{i}^{-2}\right)$, $i \in \mathbb{Z}_{3}$. Тогда подгруппь $\widehat{G}_{i}=G_{i+1} \cap G_{i-1}$ и $G_{i}$ являются централизаторами друг друга для всех $i \in \mathbb{Z}_{3}$. Кроме того, подгруппа $\widehat{G}_{i}$ изоморфна $B$ для любого $i \in \mathbb{Z}_{3}$. Например, изоморфизм $B \rightarrow \widehat{G}_{1}$ можно задать на стандартных образующих $\sigma_{k}$ группь кос следующим образом: $\sigma_{k} \mapsto d_{3}^{k-1} u_{1} d_{3}^{1-k}\left(=d_{2}^{1-k} u_{1} d_{3}^{k-1}\right), k \geqslant 1$.

Так же, как и в случае обычных зацеплений, трехстраничный подход можно применить для ленточных зацеплений. Под ленточным зацеплением мы понимаем кусочно-линейное вложение некоторого числа колец $S^{1} \times[0,1]$ и лент Мёбиуса $S^{1} \ltimes[0,1]$ в $\mathbb{R}^{3}$. Отличие этого понятия от общепринятого понятия оснащенного зацепления состоит лишь в том, что мы допускаем неорентируемые компоненты. Каждая трехстраничная диаграмма зацепления задает ленточное зацепление по правилу: каждую арку $L \cap P_{i}$ нужно заменить на замкнутую $\varepsilon$-окрестность этой арки в $P_{i}$. Таким образом, ленточные зацепления также можно кодировать словами в 12-буквенном алфавите $\mathbb{A}$. Для ленточных зацеплений имеется полньй аналог теоремы 1 , однако соответствующая полугруппа задается другим набором соотношений. Мы не выписываем их здесь, оставляя это для более подробной работы. Вместо этого мы укажем, как вычисляется скобка Кауффмана по трехстраничной диаграмме.

Пусть $K$ - алгебра, порожденная множеством образующих $\{z\} \cup \mathbb{A}$ и соотношениями:

$$
\begin{gathered}
z x=x z \quad \forall x \in \mathbb{A}, \\
a_{i}=z^{3} a_{i+1} d_{i-1}, \quad z^{3} b_{i}=a_{i-1} c_{i+1}, \quad z^{3} c_{i}=b_{i-1} c_{i+1}, \quad d_{i}=z^{3} a_{i+1} c_{i-1}, \\
b_{3} b_{2} b_{1}=z^{3} \\
b_{i} d_{i}=d_{i} b_{i}=1, \\
v w=w v, \\
\text { где } v \in\left\{a_{i-1} b_{i-1}, d_{i-1} c_{i-1}, b_{i+1} d_{i-1} d_{i+1} b_{i-1}\right\}, w \in\left\{a_{i}, b_{i}, c_{i}, b_{i-1} d_{i} d_{i-1}\right\}, \\
b_{i-1} b_{i}+z d_{i+1}+z^{2} c_{i} a_{i-1}=0,
\end{gathered}
$$

$i \in \mathbb{Z}_{3}$. Для слова $w$ в алфовите $\{z\} \cup \mathbb{A}$ мы обозначаем через $\langle w\rangle$ соответствующий элемент алгебры $K$.

ТЕорема 3. Для любого ленточного зацепления $L$ в алгебре $K$ имеет место равенство $\left\langle w_{L}\right\rangle=-\left(z^{4}+z^{-4}\right) \cdot\langle L\rangle\left(-z^{2}\right)$, где $\langle L\rangle$ есть скобка Кауффмана ленточного зачепления $L$.

Вернемся теперь к обычным (не ленточным) зацеплениям. Трехстраничное представление позволяет построить чрезвычайно эфффективньй частичньй алгоритм распознавания тривиально-

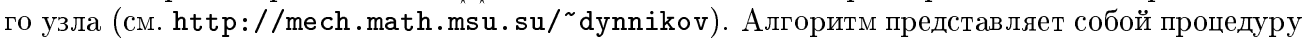
упрощения трехстраничной диаграммы узла (сложность трехстраничной диаграммы $L$ определяется числом $n(L)$ точек пересечения $L$ с линией переплета $l$ ) с помощью преобразований $L \rightarrow L^{\prime}$, описанных в следующем предложении.

ПреДЛОЖениЕ. Пусть $L$ и $L^{\prime}-$ две трехстраничные диаграммы зацепления, и для всех $i \in \mathbb{Z}_{3}$ выбраны: $\gamma_{i}$ - некоторая незамкнутая связная компонента пересечения $L \cap\left(P_{i-1} \cup P_{i+1}\right), \gamma_{i}^{\prime}$ - некоторая незамкнутая связная компонента пересечения $L^{\prime} \cap$ $\left(P_{i-1} \cup P_{i+1}\right)$ maкuе, ито $\gamma_{i} \cap \gamma_{i+1}=\varnothing, \gamma_{i}^{\prime} \cap \gamma_{i+1}^{\prime}=\varnothing, \partial \gamma_{i}=\partial \gamma_{i}^{\prime}$ для всех $i \in \mathbb{Z}_{3}$. Предположим, что в $\mathbb{Y}$ существует сохраняющая границу изотопия от $L \backslash\left(\gamma_{1} \cup \gamma_{2} \cup \gamma_{3}\right)$ $\kappa L^{\prime} \backslash\left(\gamma_{1}^{\prime} \cup \gamma_{2}^{\prime} \cup \gamma_{3}^{\prime}\right)$. Тогда зачепления $L$ и $L^{\prime}$ изотопны в $\mathbb{R}^{3}$.

Московский государственньй 